\title{
Nueva teoría sobre la depresión: un equilibrio del ánimo entre el sistema nervioso y el inmunológico, con regulación de la serotonina-quinurenina y el eje hipotálamo-hipófiso-suprarrenal
}

\author{
Leslie Alejandra Ramírez¹, Elsy Arlene Pérez-Padilla², Francisco García-Oscos³, \\ Humberto Salgado ${ }^{1}$, Marco Atzori ${ }^{4,5}$, Juan Carlos Pineda ${ }^{1}$ \\ 1 Departamento de Neurociencias, Centro de Investigaciones Regionales “Dr. Hideyo Noguchi”, Universidad \\ Autónoma de Yucatán, Mérida, México \\ 2 Facultad de Medicina, Licenciatura en Rehabilitación, Universidad Autónoma de Yucatán, Mérida, México \\ 3 Deparment of Neuroscience, University of Texas, Dallas, TX, USA \\ 4 Laboratorio de Neurobiología del Estrés, Facultad de Ciencias, Universidad Autónoma de San Luis Potosí, \\ San Luis Potosí, México \\ 5 School of Behavioral and Brain Sciences, University of Texas at Dallas, Richardson, TX, USA
}

La hipótesis sobre las causas de la depresión basada en la acción de la serotonina y del sistema inmunológico, propone que ciertos tipos de estrés distorsionan la relación entre la actividad del sistema inmunitario innato y la del sistema nervioso central.

El estrés causado por una infección o el estrés psicológico excesivo activan receptores de tipo toll, como el TLR-4, el factor de transcripción NF-kB, el inflamasoma NLRP3, así como la secreción de interleucina 1 beta (IL-1ß) e interleucina 6 (IL-6); esto causa, en primer lugar, los síntomas generales de enfermedad que aparecen con cualquier infección, pero también los síntomas característicos de la depresión como disforia y anhedonia.

Las evidencias indican que, si el estímulo persiste o se repite en las siguientes 24 horas, se activa la enzima indolamina 2,3-dioxigenasa (IDO) de la vía metabólica de la quinurenina, lo cual incrementa la síntesis del ácido quinolínico y reduce la síntesis de serotonina. El ácido quinolínico activa los receptores de N-metil-D-aspartato (NMDA) en el sistema nervioso central y estimula la secreción de, entre otras, las interleucinas IL-6 e 1L-1 $\beta$, las cuales promueven la hiperactividad del eje hipotálamohipófiso-suprarrenal y refuerzan la desviación del metabolismo del triptófano hacia la producción de ácido quinolínico, así como de las interleucinas de la inmunidad innata, con lo cual se reduce más la síntesis de serotonina y se consolida el proceso depresivo.

Este proceso puede ser iniciado por las interleucinas estimuladas por una infección, así como por algunas vacunas o por un estrés psicológico excesivo que active el eje hipotálamo-hipófiso-suprarrenal simultáneamente con la respuesta inmunológica innata, con lo que se provocaría un proceso de inflamación estéril en el sistema nervioso central.

Palabras clave: depresión; sistema nervioso; sistema inmunológico; serotonina; inmunidad innata, interleucina-1beta; interleucina-6; interleucina-10; interferón gamma; neuroglia; sistema hipotálamohipófiso-suprarrenal.

doi: https://doi.org/10.7705/biomedica.v38i3.3688

A new theory of depression based on the serotonin/kynurenine relationship and the hypothalamicpituitary-adrenal axis

The serotonergic and immunological hypothesis of depression proposes that certain types of excessive stress distort the relationship between the activities of the innate immune and central nervous systems, so that the stress caused by an infection, or excessive psychological stress, activate toll-like receptors such as the TLR-4, the transcription factor NF-kB, the inflammasome NLRP3, as well as the secretion of interleukin-1 beta (IL-1ß), interleukin-6 (IL-6) and other factors of the innate immune response, causing first, the general symptoms of the disease which appear with any infection, but also those characteristic of depressive illness such as dysphoria and anhedonia.

The evidence indicates that, if the stimulus persists or recurs within 24 hours, the indole-2, 3-dioxygenase enzyme (IDO) of the kynurenine metabolic pathway, which increases the synthesis of quinolinic acid, is activated with an associated reduction of serotonin synthesis. Quinolinic acid

\section{Contribución de los autores:}

Juan Carlos Pineda: propuesta y dirección del proyecto

Francisco García-Oscos y Humberto Salgado: experimentos y análisis de datos

Leslie Alejandra Ramírez, Elsy Arlene Pérez-Padilla, Humberto Salgado, Marco Atzori y Juan Carlos Pineda: discusión y escritura de la revisión 
activates NMDA receptors in the central nervous system and stimulates the secretion of interleukins IL-6 and $1 \mathrm{~L}-1 \beta$, among others, promoting hyper-activity of the HPA axis and reinforcing a bias of the tryptophan metabolism to produce quinolinic acid, and interleukins by the innate immune system, further reducing the synthesis of serotonin and consolidating the depressive process.

We discuss the evidence showing that this process can be initiated by either interleukin stimulated by an infection or some vaccines or excessive psychological stress that activates the HPA axis together with said innate immune response, causing a process of aseptic inflammation in the central nervous system.

Key words: Depression; nervous system; immune system; serotonin; immunity, innate; interleukin1 beta; interleukin-6; interleukin-10, interferon gamma; neuroglia; pituitary-adrenal system.

doi: https://doi.org/10.7705/biomedica.v38i3.3688

El sistema inmunológico y el sistema nervioso comparten las funciones de reconocer objetos, discernir sus cualidades (si son propios o extraños; inocuos o peligrosos) y de generar una respuesta adaptativa relacionada con ellos.

Ambos sistemas desarrollaron reacciones al estrés ambiental relativamente simples y rápidas. Por ejemplo, el sistema nervioso desarrolló el arco reflejo, en tanto que el sistema inmunitario reacciona con la inflamación y el 'reflejo antiinflamatorio' $(1,2)$. Los dos sistemas expresan reacciones de vigilancia celular destinadas a detectar invasores potencialmente peligrosos presentes en todos los organismos multicelulares (3). Tanto el arco reflejo del sistema nervioso como el reflejo inflamatorio del sistema inmunitario operan mediante respuestas rápidas e innatas (1).

El reflejo inflamatorio depende de la detección de patrones moleculares específicos presentes en los invasores pero no expresados por los tejidos propios. Esto ha resultado tan eficaz que los principales componentes de dicha inmunidad innata están conservados, como la familia de los receptores de tipo toll (Toll-like receptors, TLR) y los inflamasomas, tanto en las plantas como en los mamíferos (4).

\section{Inmunidad innata y sistema nervioso}

El sistema inmunitario se clasifica según el tipo de respuesta: i) una innata, rápida, que produce inflamación como mecanismo de defensa primario, con especificidad limitada, y ii) una adaptativa más lenta, pero más específica. La inmunidad innata (figura 1) se activa mediante receptores en la membrana extracelular, entre los cuales se

\section{Correspondencia:}

Juan Carlos Pineda, Centro de Investigaciones Regionales "Dr. Hideyo Noguchi", Universidad Autónoma de Yucatán, Av. Itzaes $N^{\circ} 490 \times 59$, Mérida, Yucatán, México

Teléfono: (52 9991) 638 169; fax: (52 9999) 236120

pcortes@correo.uady.mx; jcpincor@gmail.com

Recibido: 12/01/17; aceptado: 14/12/17 encuentran los de la familia TLR, en tanto que la respuesta inmunitaria aprendida reconoce antígenos muy específicos contra casi cualquier patógeno (1).

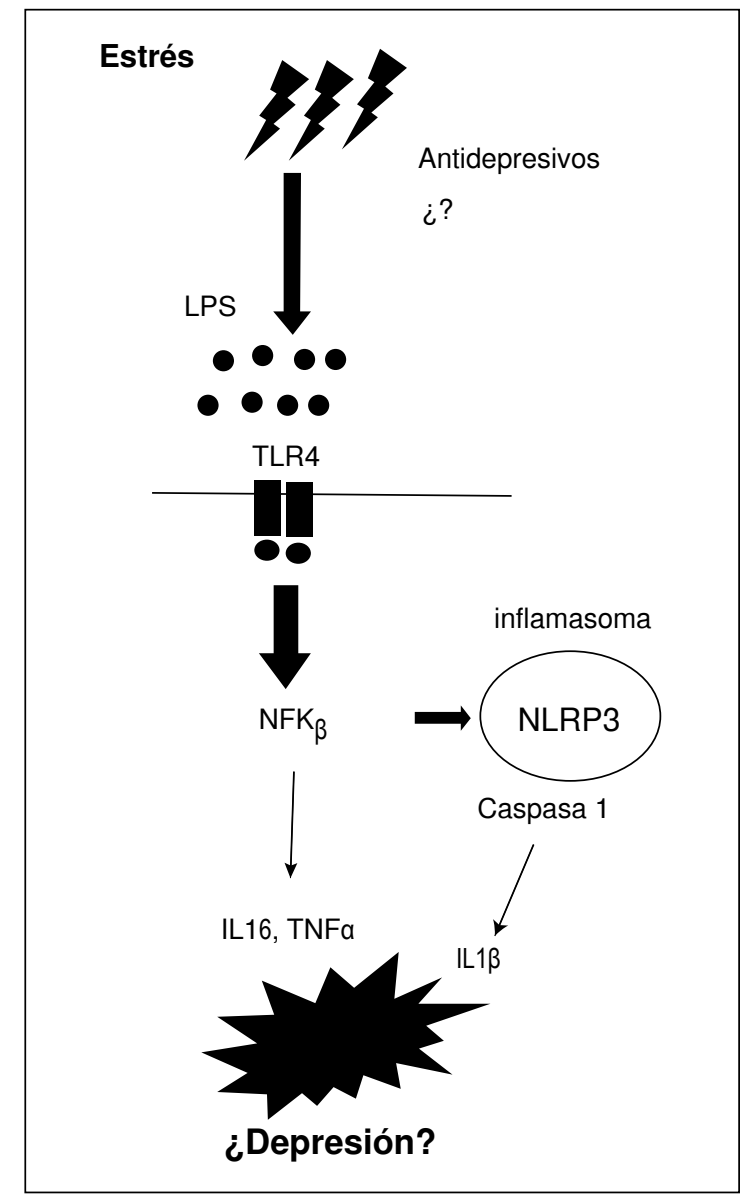

Figura 1. El estrés provocado por la aplicación de lipopolisacáridos de la pared celular de bacterias Gram negativas o por otras causas, activa la respuesta inmunitaria innata y los receptores de tipo toll en la membrana de diversas clases de linfocitos. La activación de los receptores TLR4 induce la activación de la subunidad $\beta$ del factor necrótico kappa que, a su vez, activa el inflamasoma NLPR3, lo que provoca la liberación de la caspasa 1 y la subsecuente liberación de IL-1b e IL-6. Estas citocinas median la inflamación, los signos generales de enfermedad y, posiblemente, la depresión. 
En la figura 1 se ilustran los principales componentes de la inmunidad innata que, como ya se dijo, están muy conservados tanto en plantas como en animales, incluidos los mamíferos (4). El sistema nervioso de los mamíferos también puede detectar estímulos inflamatorios directamente, lo que le permite reconocer fuentes potenciales de daño utilizando el dolor como señal, así como modular la respuesta a la infección $(1,2)$. Un ejemplo de la interacción directa entre el sistema nervioso y el sistema inmunitario, es el caso de la influencia de la noradrenalina y de la acetilcolina en la reacción conocida como reflejo inflamatorio, o su contraparte, el reflejo antiinflamatorio, este último producido en la llamada vía colinérgica antiinflamatoria (3).

El reflejo inflamatorio puede concebirse como el circuito de un reflejo prototípico análogo al arco reflejo del sistema nervioso y capaz de mantener una respuesta inmunitaria equilibrada, homeostática, en la cual participan ambos sistemas; en dicho reflejo, los mediadores moleculares de la inmunidad innata activan señales eléctricas aferentes en el nervio vago. Los potenciales de acción viajan por la vía aferente de este nervio al tronco del encéfalo, en donde modulan las interneuronas del núcleo del tracto solitario que, a su vez, regulan los núcleos motores del nervio vago. Los axones de las neuronas eferentes de estos núcleos descienden por el nervio vago hasta el ganglio celíaco, por donde viajan las señales eléctricas a una estación de retransmisión para propagar las señales al nervio esplénico y al bazo. La vía de señalización culmina en el bazo con la liberación del neurotransmisor acetilcolina, el cual activa los receptores nicotínicos $\alpha 7$ (nAChR) que se expresan en los macrófagos del bazo e inhiben la secreción de las citocinas (2).

Se ha podido comprobar que el reflejo inflamatorio y el antiinflamatorio modulan de manera recíproca diversos sistemas, como el cardiaco y el digestivo, así como estructuras del sistema nervioso central relacionadas con el control del ánimo $(2,3)$. Este circuito controla y modula la respuesta inmunitaria innata en un marco de tiempo específico y rápido, y puede activarse o inhibirse por acción de la modulación de diversas regiones del cuerpo donde la actividad eléctrica del nervio vago puede suprimir tónicamente la actividad de los linfocitos que participan en la respuesta inmunitaria innata, como sucede en el control neural de la frecuencia cardíaca. Otra evidencia de dicho control es la respuesta hipersensible o desinhibida frente a estímulos invasivos o infecciosos, cuando los componentes del sistema nervioso (por ejemplo, el nervio vago o el esplénico) o los componentes moleculares de la vía colinérgica antiinflamatoria, se eliminan experimentalmente o se inhiben farmacológicamente $(1,3)$.

\section{Respuesta inmunitaria innata y depresión}

Es posible que la regulación del reflejo neural en la respuesta inmunitaria innata se haya originado desde un comienzo en la evolución de la inmunidad. En un estudio del 2009, Aballay, et al., evidenciaron que en Caenorhabditis elegans, un gusano nematodo que posee un sistema inmunitario innato primitivo y un sistema nervioso evolutivamente antiguo, se expresan genes que activan la respuesta inmunitaria innata, la cual conduce a cambios específicos de comportamiento, incluida la evitación de los agentes patógenos, así como la migración para alejarse del sitio donde se encuentran olores patógenos $(5,6)$.

Según el estudio de Maier, et al., en 1998, la inflamación sistémica se correlaciona con cambios en el cerebro, independientemente del suceso iniciador (una infección, un factor de estrés psicológico o el dolor), y los síntomas o las manifestaciones conductuales de la depresión emergen impulsados por los mismos cambios en el cerebro (7). Estos autores especularon que durante la evolución surgió primero el sistema inmunitario para luchar contra los patógenos. Cuando los organismos multicelulares requirieron de un mecanismo para reaccionar ante una amenaza psicológica externa, el cuerpo recurrió al mismo sistema inmunitario ya existente.

Maes, et al., hicieron planteamientos similares y propusieron una conexión evolutiva entre los factores psicológicos estresantes y la activación inflamatoria $(7,8)$. Pace, et al., por su parte, sugirieron en un estudio del 2012 que el sistema inmunitario innato puede activarse solo por el estrés provocado por una amenaza psicológica, como la expectativa del ataque de un depredador, sin necesidad de un factor material de estrés, y que dicha característica es un factor adaptativo (9). Este fenómeno ya había sido planteado por Smith en 1991, en su teoría de los macrófagos en la depresión, en la cual postuló que las citocinas secretadas durante el estrés de una infección producían depresión (10).

Según esta propuesta, las concentraciones en sangre de los marcadores biológicos inflamatorios, como la proteína $G$ reactiva y el fibrinógeno, se incrementan en los pacientes con depresión clínica 
(10). Smith, Maes, et al., y Pace, et al., sugirieron que tanto la fase aguda de la inflamación debida a una infección como la disforia de la depresión, responden a los mismos mecanismos $(8,9,10)$. Las citocinas proinflamatorias responsables de la reacción inflamatoria en su fase aguda, también causan otras manifestaciones clínicas de depresión, incluidos la hiperactividad del eje hipotálamo-hipófisosuprarrenal, la alteración del metabolismo de la serotonina y los síntomas neurovegetativos.

\section{Objetivo de la revisión temática}

Considerando que, a pesar de haber sido formulada hace más de 20 años, la teoría inmunológica de la depresión no ha tenido una amplia difusión, en esta revisión nos propusimos describir la secuencia histórica de los principales datos comprobados y las observaciones experimentales sobre las interacciones entre el sistema inmunitario innato, el sistema nervioso central, el eje hipotálamo-hipófisosuprarrenal y la vía metabólica del aminoácido triptófano, que condujeron a enunciar dicha teoría.

La información se presenta organizada históricamente y se resalta su contribución como respaldo de la teoría inmunológica de la depresión.

\section{Inmunidad innata y sistema nervioso}

La inmunidad innata depende de la detección específica de los patrones moleculares presentes en los organismos invasores, como los que se expresan en la pared celular de las bacterias Gram negativas, pero no así en los tejidos del huésped. Los principales componentes de la inmunidad innata (figura 1) están muy conservados tanto en plantas como en animales, incluidos los mamíferos (4) y los humanos (11).

\section{La enfermedad depresiva y las citosinas}

Como ya se señaló, una de las primeras observaciones sobre la relación del sistema inmunitario con la depresión clínica fue el aumento de las concentraciones en sangre de los marcadores biológicos inflamatorios, como la proteína G reactiva y el fibrinógeno, en pacientes con depresión (10). Con base en estas observaciones, Smith presentó la teoría de los macrófagos en la depresión, en la cual propuso que la fase aguda de la inflamación causada por una infección responde a los mismos mecanismos que la anhedonia y la disforia observadas durante la depresión.

En apoyo de esta teoría, se reportó que muchos pacientes con cáncer o con hepatitis $C$ que recibían inmunoterapia habían desarrollado síntomas de depresión (12,13). La investigación sistemática de estos síntomas demostró que son causados por el tratamiento que reciben los pacientes, y reveló que estos pueden dividirse en dos categorías distintas. En la primera categoría, los signos neurovegetativos y los síntomas somáticos de depresión, los cuales son de aparición temprana y están presentes en todos los pacientes, incluyen manifestaciones parecidas a las de la gripe, como fatiga, anorexia, dolor y trastornos del sueño. En la segunda categoría se incluyen los síntomas psicológicos de la depresión, entre ellos, las alteraciones cognitivas leves y los síntomas propios del estado de ánimo depresivo, los cuales frecuentemente se acompañan de ansiedad e irritabilidad. Estos síntomas tardan más en aparecer y son experimentados hasta por la mitad de los pacientes $(14,15)$.

De hecho, cuando se examinó a los pacientes que desarrollaron los síntomas psicológicos de la depresión, se encontró que después de la primera inyección de IFN- $\alpha$ tuvieron una mejoría en el control homeostático del eje hipotálamo-hipófisosuprarrenal, lo que evidenció que la vulnerabilidad frente a la depresión inducida por la inmunoterapia involucra factores tanto fisiológicos como de riesgo psicológico $(14,15)$. En estos pacientes, el tratamiento previo con paroxetina, un antidepresivo tricíclico e inhibidor selectivo de la recaptura de serotonina, redujo los síntomas psicológicos, pero tuvo poco efecto sobre la sintomatología neurovegetativa (14). Por otro lado, el antidepresivo tricíclico imipramina redujo los índices depresivos y disminuyó la inflamación producida por el estrés (16).

Estas observaciones sugerían que la depresión inducida por la inmunoterapia efectivamente podría ser producto de un proceso inflamatorio en pacientes vulnerables $(17,18)$, dado que la administración sistémica o central de un antagonista soluble del receptor IL-1, el IL-1ra, es capaz de revertir los cambios conductuales inducidos en un modelo animal de depresión producido imponiendo el aislamiento social a los roedores. Como se verá en las siguientes secciones, estos resultados se han podido reproducir ampliamente (19).

En este contexto surgió la siguiente pregunta: ¿es la respuesta inmunológica a los agentes infecciosos el origen de la depresión? Para responderla, se estudió la relación entre los síntomas que la reacción inmunitaria innata produce cuando hay infección, y aquellos de la depresión $(18,19)$. 


\section{Síntomas generales de enfermedad y síntomas de depresión}

Los síntomas generales de enfermedad constituyen un conjunto coordinado de cambios conductuales que se verifica en individuos enfermos durante el curso de una infección $(18,20)$. Algunos de ellos producen una sensación de malestar general y desinterés por las actividades placenteras, que se asemeja a la anhedonia. La disforia, o ánimo decaído, y la anhedonia son los dos síntomas específicos que caracterizan a los trastornos depresivos, pero también forman parte de la reacción individual al estrés ambiental y son controlados por la respuesta inmunitaria de la vía celular o la humoral y, por consiguiente, aparecen en múltiples enfermedades (21).

En roedores se ha demostrado que la administración sistémica o central de las citocinas IL-1 $\beta$ o IL6 , o del TNF- $\alpha$ induce el espectro completo de los síntomas generales de enfermedad, dependiendo de la dosis y la duración de la administración (22). En general, los animales inyectados con estas citocinas permanecen en un rincón de su jaula, en una postura encorvada, y muestran poco o ningún interés en su entorno físico y social a menos que sean estimulados. Específicamente, expresan disminución de la actividad motora, aislamiento social, reducción de la ingestión de alimentos y agua, alteración de la cognición y, a menudo, aumento de la sensibilidad al dolor (23), síntomas estos que se observan también durante la depresión. Además, en los animales sometidos a estos protocolos, también se registró aumento de los índices depresivos en las pruebas conductuales que miden la depresión, como el tiempo de inmovilidad en la prueba de nado forzado y el volumen de ingestión de solución con sacarosa (23). Sin embargo, no todo concuerda con lo observado en pacientes con depresión, pues los animales también presentaron un aumento del sueño de ondas lentas, lo cual no sucede en los casos humanos (24).

\section{Respuesta inmunitaria innata en el sistema nervioso periférico}

La respuesta inmunitaria innata periférica controla el cerebro mediante varios medios que actúan en paralelo. Una primera ruta involucra los nervios aferentes del sistema nervioso autónomo. Las citocinas producidas localmente activan los nervios aferentes primarios del nervio vago durante una infección abdominal o visceral (25) o, el nervio trigémino, durante una infección en boca y lengua (26). La segunda es la ruta humoral, la cual activa los TLR en células similares a los macrófagos que residen en los órganos circunventriculares. Entre estos, los macrófagos asociados con el plexo coroideo reaccionan a los patrones moleculares asociados con agentes patógenos de la circulación, mediante la producción de citocinas proinflamatorias $(27,28)$. Una tercera vía incluye los transportadores de citocinas en la barrera hematoencefálica: las citocinas proinflamatorias entran en la circulación sistémica y, desde ahí, pueden tener acceso al cerebro mediante estos sistemas de transporte (29). Por último, está la ruta que involucra los receptores de IL- $1 \beta$ e IL- 6 , que se encuentran en los macrófagos perivasculares y las células endoteliales de las vénulas del cerebro (30).

El acoplamiento de estas vías de comunicación del sistema inmunitario al cerebro conduce a la producción de citocinas proinflamatorias por parte de las células microgliales mediante la acción convergente de dos eventos con diferentes cursos de tiempo: uno que lleva a la activación de la vía neural aferente rápida y otro de propagación más lenta que lleva el mensaje de las citocinas dentro del cerebro. La activación de la vía neural, probablemente, sensibiliza estructuras cerebrales blanco para la producción y la acción de las citocinas que se propagan desde los órganos circunventriculares y el plexo coroideo en el cerebro $(16,31)$.

En este sentido, se ha propuesto que el cerebro forma una 'imagen' de la respuesta inmunitaria periférica innata, cuyos componentes moleculares primarios son similares a los de la reacción descrita en la periferia, sin que la formación de esta 'imagen inmunológica' en el cerebro implique una invasión de las células inmunitarias en el parénquima cerebral, lo que indica que las alteraciones no se deben al daño tisular producido directamente por la infección $(29,30,32)$.

Sin embargo, no se sabe aún cuáles son los circuitos del cerebro que median las diversas acciones del comportamiento depresivo causado por estrés o los síntomas generales de enfermedad provocados por las citocinas. Por ejemplo, no es probable que los síntomas generales de enfermedad y el aislamiento social inducidos por las citocinas, estén mediados por las mismas áreas del cerebro que producen la reducción del consumo de alimentos o la activación del eje hipotálamohipófiso-suprarrenal (33), o la disrupción del control ejecutivo orquestado por la corteza prefrontal $(34,35)$. La conducta en los modelos de depresión, la cual se ha relacionado con las células de la capa 
granular del giro dentado, de la capa de células piramidales del hipocampo y de la hipófisis (36), es influenciada por los receptores de las citocinas IL-1 $\beta$ e IL-6 $(30,34,37,38)$.

En trabajos previos de nuestro grupo, se ha evidenciado que la IL-6 es capaz de modular las corrientes inhibitorias GABA-érgicas producidas por la estimulación de las fibras recurrentes en la corteza prefrontal o auditiva de la rata $(34,35)$. Recientemente, mediante registros intracelulares se encontró, en la preparación del corte de cerebro mantenido en condiciones in vitro, que esta interleucina también puede modular la influencia de la norepinefrina sobre las corrientes inhibitorias posinápticas (Inhibitory Postsynaptic Current, IPSC) en la segunda y la tercera capa de la corteza auditiva primaria (A1) de roedores.

En la figura $2 A$ se evidencia la influencia de la norepinefrina en dichas corrientes inhibitorias GABAérgicas posinápticas registradas en neuronas piramidales de la segunda y la tercera capa de la corteza auditiva de ratón. Como ya se había observado, la norepinefrina $(20 \mu \mathrm{M})$ incrementó de manera reversible la amplitud de tales corrientes inhibitorias (39). Este resultado sugirió que la sensibilidad auditiva del ratón podría incrementarse bajo la influencia de esta amina biogénica. Sin embargo, en el presente trabajo se pudo demostrar que la activación de la inmunidad innata por inflamación cambia radicalmente esta relación.

En otro experimento (figura 2B), se demostró la acción de la IL-6 en las mismas corrientes con la aplicación de $10 \mathrm{ng} / \mathrm{ml}$ de IL-6 directamente en una neurona piramidal de la segunda y la tercera capa de la corteza auditiva. En la figura se ven las corrientes inhibitorias antes y después de la aplicación de los $10 \mathrm{ng} / \mathrm{ml}$ de IL-6.

Como se aprecia, la aplicación de la interleucina produjo una reducción de su amplitud, de aproximadamente el $50 \%$ (figura $2 \mathrm{~B}$ ). Sin embargo, cuando se aplicó norepinefrina en presencia de la IL-6, ya no se produjo ningún cambio en las corrientes sinápticas inhibitorias, lo que sugiere que, cuando la inmunidad innata se activa, la norepinefrina pierde la modulación positiva sobre las corrientes inhibitorias que sí se produce en condiciones de control, lo cual contribuye a incrementar la excitabilidad del sistema (figura 2B). Aun cuando estos resultados no implican necesariamente cambios en la respuesta conductual, concuerdan con las modificaciones observadas en dicha reacción con la aplicación de la citosina IL-6 $(34,39)$.

\section{Receptores de tipo toll 4 y citocinas IL- $1 \beta$ e IL- 6 en la depresión}

Además de la citocina IL-1 $\beta$ y la IL-6, que con frecuencia se han relacionado con la depresión, también se han encontrado relaciones con otras moléculas y receptores de la respuesta inmunitaria innata, en particular los receptores de tipo toll 4 (TLR4) (13). La activación de tales receptores desencadena procesos de transcripción proinflamatorios mediante proteínas adaptadoras que inducen la transcripción de los factores NF-kappa B, AP-1 e IRF3 $(40,41)$. A su vez, la activación de estos factores de transcripción promueve la de la producción de citocinas proinflamatorias, incluidas la IL-1 $\beta$, el TNF- $\alpha$, la IL-6, y la CXCL10, así como el incremento de proteínas como la ciclooxigenasa 2 (COX-2), lo que resulta en un aumento de la señalización proinflamatoria observada en el paradigma de choques eléctricos inevitables en roedores, en el modelo del fenómeno de desesperanza aprendida $(42,43)$.

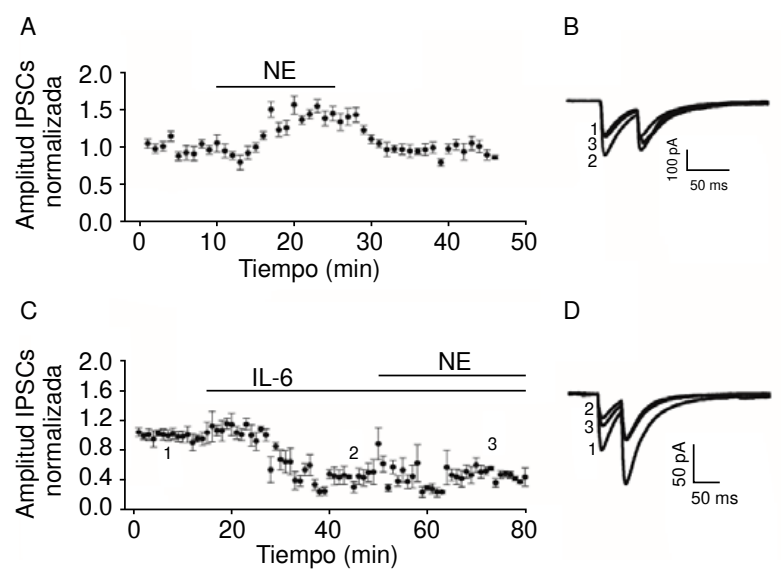

Figura 2. La citosina IL-6 bloquea la influencia de la noerepinefrina en las corrientes sinápticas inhibitorias (IPSC) en neuronas piramidales de la capa 2-3 del área A1 en la corteza temporal de la rata. A. Curso temporal de la amplitud de las IPSC mediante la estimulación de las fibras aferentes cercanas al sitio de registro antes y después de la aplicación de norepinefrina $(20 \mathrm{mM})$ directamente en el baño. B. IPSC sobrepuestas en el punto indicado por los mismos números en A durante este experimento. C. Como en A, curso temporal de la amplitud de las IPSC antes y después de la aplicación de IL-6 recombinante de rata $\left(10 \mathrm{ng} / \mathrm{ml}\right.$; $R$ \& D Systems ${ }^{\circledR}$, Minneapolis, Minnesota) y de norepinefrina $(20 \mu \mathrm{M})$ en presencia de la IL-6 (aplicación directamente en el baño). D. Como en B, las IPSC sobrepuestas en el punto indicado por los mismos números durante el experimento mostrado en C. La IL-6 fue activada en una solución salina amortiguada con fosfato en una concentración de 0,5 g/1 ml. La norepinefrina $(20 \mu \mathrm{M})$ se aplicó iontoforéticamente en el sitio de registro. Los puntos indican el promedio de la amplitud de las IPSC. 
Una diferencia notable entre el fenómeno producido por el aumento de la señalización de las neurocininas y el de la neuroinflamación, y la depresión mayor, es la reversibilidad de los efectos. El aumento de citocinas centrales resultante de infecciones que no son graves, del ejercicio o del estrés moderado, produce cambios neuronales reversibles (44). Estos cambios neuronales provocados por las citocinas IL-1 $\beta$, IL- 6 y TNF- $\alpha$, incluyen la estimulación de los receptores de NMDA y del ácido a-amino-3-hidroxi-5-metilo-4isoxazolpropiónico (AMPA), y el equilibrio de su activación, así como la disminución de la expresión de los receptores GABA en las neuronas, lo que provoca un aumento de la excitabilidad reversible (45). En la enfermedad depresiva, a esto se suma la activación del sistema serotoninérgico pasadas las 24 horas; además, estos cambios pueden continuar durante semanas, meses o años (44).

\section{El metabolismo del triptófano y la serotonina y la depresión}

Uno de los mediadores moleculares clave en la inducción del desarrollo de los síntomas depresivos en los seres humanos después de la administración de la citosina IFN- $\alpha$, es la enzima indolamina 2,3-dioxigenasa (IDO), que convierte el triptófano en quinurenina, un precursor de la neurotoxina 3-hidroxiquinurenina, o en ácido quinurénico, un metabolito neuroprotector (46) (figura 3).

De hecho, la producción de metabolitos de triptófano neurotóxicos después de la activación de la IDO, se considera un paso esencial en el proceso fisiopatológico de la depresión, lo cual se suma al hecho de que durante el estrés se reduce la síntesis del 5-hidroxitriptofano $(5-\mathrm{HT})$ en el cuerpo y la de la serotonina en el cerebro (46). La activación de la IDO lleva a la producción de 3-hidroxiquinurenina y ácido quinolínico, los cuales producen síntomas de la depresión análogos a los síntomas generales de enfermedad. Dichos metabolitos son agonistas de los receptores NMDA y potencialmente neurotóxicos, en tanto que el ácido quinurénico es un antagonista de dichos receptores y, generalmente, se considera neuroprotector (47-50) (figura 3).

Las citocinas proinflamatorias incrementan la enzima quinurenina-3-mono-oxigenasa, la cual degrada la quinurenina en la 2-hidroxiquinurenina y, de este modo (figura 3), desvían la vía de quinurenina hacia la formación de dichas sustancias neurotóxicas. O'Connor, et al., demostraron que la administración periférica de IDO inducía un estado de depresión. El bloqueo de su activación directamente con un antagonista de dicha activación, o indirectamente con fármacos antiinflamatorios, impide la aparición del comportamiento depresivo porque atenúa la expresión de citocinas proinflamatorias inducidas por lipopolisacáridos que activan la inmunidad innata $(32,51,52)$.

En línea con estas observaciones, cuando se produjo el modelo de depresión mediante la aplicación de lipopolisacáridos en roedores, aumentó la expresión de IDO en el cerebro 24 horas después de la inyección (53), tiempo que coincide con el de la aparición del comportamiento depresivo (54). El aumento de la actividad enzimática de la IDO se asoció con un aumento en la relación entre quinurenina y triptófano en la periferia y en el cerebro, procesos que ocurren también 24 horas después de la aplicación de los lipopolisacáridos $(55,56)$. Los análogos de los lipopolisacáridos producen el mismo efecto en el cerebro de la rata (57).

En las ratas que recibieron interferón alfa recombinante humano, el índice depresivo (tiempo de inmovilidad) se incrementó en la prueba de nado forzado. Sin embargo, su aplicación no incrementó la relación entre quinurenina y triptófano en el cerebro. El antidepresivo tricíclico imipramina bloqueó los efectos conductuales del interferón, pero no

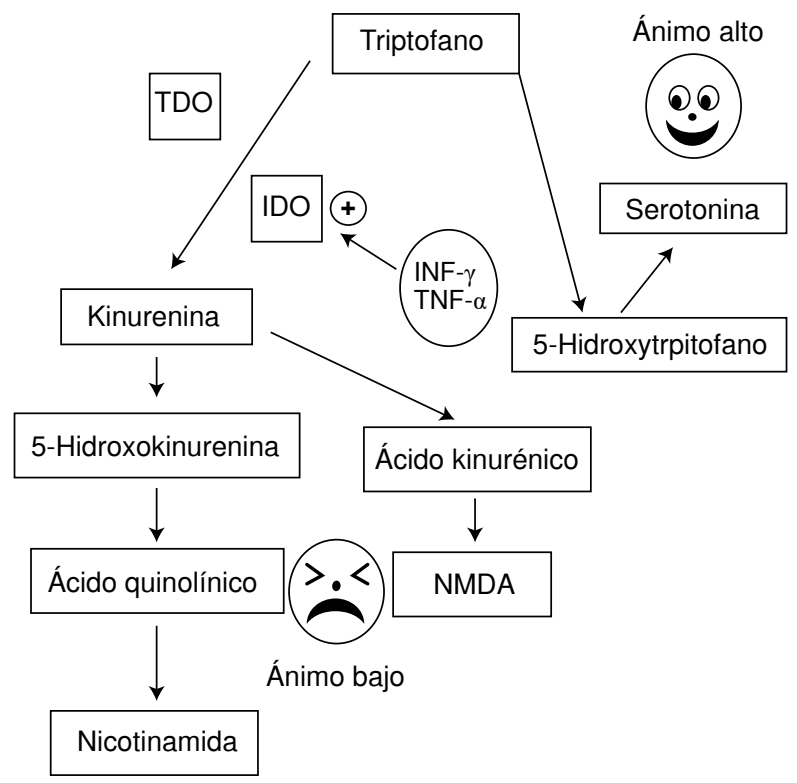

Figura 3. El metabolismo del triptófano puede incrementar la actividad de la enzima triptófano hidroxilasa, aumentando las concentraciones del 5-hidroxitriptofano y promoviendo un estado eutímico del sistema, o incrementar la actividad de la enzima indolamina 2,3-dioxigenasa (IDO) y las concentraciones de ácido quinolínico promoviendo un estado depresivo. 
tuvo efecto sobre dicha relación (58). En apoyo de la hipótesis de que la activación del sistema inmunitario innato produce trastornos del ánimo, la supresión del gen IDO-1, que codifica para la IDO, abolió el desarrollo del comportamiento depresivo inducido por la vacuna contra la tuberculosis (BCG) (59).

\section{Eje hipotálamo-hipófiso-suprarrenal en la inflamación y en la depresión}

Se ha demostrado que el estrés crónico y la activación de las vías proinflamatorias se acompañan de resistencia de los receptores a los glucocorticoides (60). Por ejemplo, en un estudio de monocitos de sangre periférica de los cuidadores de pacientes con tumor cerebral maligno, se evidenció que el estrés producía resistencia funcional a los glucocorticoides, liberando la activación de las vías de control proinflamatorio de la transcripción (61). En otro estudio, la exposición recurrente a los estímulos estresantes aumentó significativamente la expresión del ARN mensajero y los niveles de proteínas plasmáticas de la IL-1 $\beta$. Por último, los ratones que carecían del receptor IL-1 $\beta$ exhibieron hipertrofia suprarrenal y elevación de los niveles séricos de corticosterona como reacción al estrés (52).

También se ha reportado repetidamente que la concentración de la hormona cortisol se encuentra elevada en las personas deprimidas $(62,63)$. De hecho, la falta de inhibición de la hormona liberadora de corticotropina de las neuronas parvocelulares en el núcleo paraventricular del hipotálamo, es la causa inmediata de las elevaciones de cortisol (52). Una vez activado, el hipocampo se comunica con el núcleo paraventricular del hipotálamo a través de conexiones en el núcleo ventral de la estría terminal (BNST), e inhibe la liberación de corticotropina. EI BNST es probablemente un núcleo crucial en la regulación de la liberación de corticotropina en situaciones de estrés crónico (64-66).

\section{Eje hipotálamo-hipófiso-suprarrenal e inflamación}

La inflamación inducida podría estimular la actividad del eje hipotálamo-hipófiso-suprarrenal por medio de la acción directa de las citocinas en el cerebro, o mediante la inducción de resistencia a los glucocorticoides (67). De hecho, la hiperactividad es un marcador de la resistencia a los glucocorticoides e indica que la acción de las hormonas gluco-corticoides en los tejidos blanco resulta ineficaz, lo cual podría conducir a la activación del sistema inmunitario. Existe gran interés por encontrar la causa de esta alteración (67).

En diversos modelos animales de la depresión, se ha evidenciado que esta se asocia con una disminución de la expresión de los glucocorticoides en el cerebro de fenotipo depresivo (67-69). En los humanos, dicha privación aumenta el riesgo de depresión. Por el contrario, un ambiente de seguridad temprana provocó un incremento en la expresión de dichos receptores en el hipocampo, así como disminución de la propensión a la depresión en la edad adulta (69). Los resultados de estos estudios sugirieron que las circunstancias en los primeros años de vida modulan la expresión de los receptores de glucocorticoides en el hipocampo, lo que, a su vez, puede modular los comportamientos relacionados con el estrés en la edad adulta. Aunque estos estudios no proporcionan una relación causal directa entre la expresión de los glucocorticoides y los comportamientos relacionados con la depresión, en las pruebas de correlación constituyen una clara evidencia de que las alteraciones en su actividad serían un factor importante en la etiología de la depresión (67).

En ratones modificados genéticamente con la inactivación específica de la señalización de los receptores de tipo toll en el hígado y en la médula ósea, se encontró que, durante la inflamación sistémica, se produce una modulación cruzada del sistema inmunitario y el suprarrenal que afecta la actividad del eje hipotálamo-hipófiso-suprarrenal (70). En algunos estudios, se ha demostrado que la señalización de los TLR4 es capaz de estimular el eje hipotálamo-hipófiso-suprarrenal (71), y que su activación es suficiente para causar la liberación de glucocorticoides de las células suprarrenales (72). Además, pueden causar un incremento en la expresión del gen $\mathrm{CRH}$ en las neuronas paraventriculares del hipotálamo (73), así como aumento de la corticotropina en el suero. Los protocolos conductuales que producen estrés crónico variable o estrés por derrota social, pueden sensibilizar el eje hipotálamo-hipófiso-suprarrenal y la respuesta inmunitaria a la exposición posterior a los lipopolisacáridos, e inducir diferencialmente respuestas incrementadas a las neurocininas y a las citocinas periféricas (74).

\section{Glía y depresión}

Como lo señalaron Raison, et al., las células gliales están implicadas tanto en la fisiopatología de la depresión mayor como en la acción de los 
antidepresivos. En diversos estudios, se han abordado las alteraciones en la morfología y en el número de astrocitos, microglías y oligodendrocitos durante el proceso depresivo en humanos y en modelos animales de la depresión (17). Estas observaciones se han vinculado a pruebas funcionales de las alteraciones en el sistema de las células gliales y de las neuronas, los cambios en la concentración de los neurotransmisores o sustancias inmunoactivas o en el estado de mielinización, así como en la formación de las sinapsis (75).

Se ha evidenciado, además, una relación causal entre la disfunción de los astrocitos y la depresión, demostrada, por ejemplo, porque la destrucción selectiva de los astrocitos frontocorticales mediante la toxina selectiva del ácido L-a gliotoxinaaminoadípico, es suficiente para desencadenar la depresión (76). Asimismo, el fenotipo de tipo depresivo también apareció debido a la inhibición funcional de los astrocitos mediante la remoción de su sinaptobrevina-2, o al bloqueo de la liberación vesicular (77). Además, se ha reportado que diversas alteraciones moleculares en los astrocitos producen signos de depresión, como la alteración de la conexina 43, que participa en uniones comunicantes (78), o el bloqueo del receptor IP3 de tipo 2 (77), o la acuaporina-4 (79). Los tratamientos que alivian los síntomas depresivos, como los inhibidores selectivos de la recaptación de la serotonina (80), la terapia electroconvulsiva o la estimulación cerebral profunda (81), pueden recuperar la función de los astrocitos (82).

Las observaciones sobre lo que ocurre en el proceso depresivo indican que la actividad neuronal, lejos de actuar de manera aislada, lo hace en estrecha interacción con el sistema inmunitario y el sistema nervioso por medio de los astrocitos y de las microglías, que interactúan estrechamente en la actividad sináptica integrando la estructura llamada sinapsis 'cuadripartita' (del término quadpartite synapse). A diferencia de la descripción clásica de los dos componentes, previos y posteriores a la sinapsis, en la actualidad se reconocen cuatro componentes: la célula presináptica, la célula posináptica y la participación estrecha de los astrocitos y la microglía (83).

El hallazgo de la capacidad de la glía para influir en la neurotransmisión serotoninérgica ilustra cómo no se trata simplemente de reemplazar antiguas concepciones sobre la causa de los síntomas depresivos (como la alteración del metabolismo de la serotonina), sino de complementar la teoría con la participación de los mecanismos neuroinmunitarios y ampliar la explicación de los mecanismos neurales de la enfermedad. Se ha confirmado, por ejemplo, que la administración de glucocorticoides emuló el estrés inducido por la sensibilización inmunológica (84), efecto que fue bloqueado por un antagonista de los receptores de glucocorticoides, lo que indicaría que la señalización de estos receptores también sería un factor esencial en la génesis de la depresión mayor (84).

\section{Teoría de la depresión por la traducción de la señal social}

Actualmente, el protocolo conductual para simular la enfermedad depresiva en roedores usado con más frecuencia se conoce como "protocolo de derrota social". Slavich, et al., propusieron la teoría "de depresión por la traducción de la señal social", en la cual se enmarca el protocolo conductual mencionado.

Esta teoría propone que las situaciones que impliquen amenaza social se representan neurológicamente en regiones del cerebro, como la ínsula anterior y la región dorsal de la corteza cingulada anterior. Ambas regiones procesan las experiencias de afecto negativo o las situaciones que se detectan como un error insoluble $(85,86)$. Aunque estas regiones no regulan directamente la actividad inflamatoria, tienen conexiones anatómicas con regiones del cerebro de nivel inferior, incluidos los núcleos de control autonómico del hipotálamo y el tronco cerebral, los cuales influyen en la inflamación sistémica mediante la modulación de la actividad del eje hipotálamo-hipófiso-suprarrenal y el sistema nervioso simpático (87).

La producción de cortisol asociada con la activación del eje hipotálamo-hipófiso-suprarrenal, suprime la actividad inflamatoria, en tanto que la producción de epinefrina y norepinefrina relacionada con el sistema nervioso simpático, promueve la inflamación mediante la interacción con las células inmunitarias para iniciar la activación de los factores de transcripción NF-kB y AP -1 . Estas interacciones desvían la vía metabólica del triptófano hacia la activación de la enzima IDO2 , e inducen la activación de los factores NF-kB y AP-1 que regulan la expresión de genes de la respuesta inmunitaria proinflamatoria, incluidas la IL-1 $\beta$, la IL6 y el TNF. Una vez activados, los genes asociados producen secuencias de aminoácidos que forman la base para diferentes proteínas, como las citocinas proinflamatorias IL-1 $1 \beta$, IL- 6 y TNF- $\alpha$ (87). 
El aumento de la actividad inflamatoria induce, a su vez, algunos de los síntomas somáticos, vegetativos, cognitivos y afectivos de la depresión, cuyos efectos son mediados por varias vías que no son mutuamente excluyentes.

\section{Conclusión}

Se ha descrito cómo la actual teoría de la depresión basada en la actividad de la serotonina y del sistema inmunitario incorpora los factores propuestos en hipótesis previas, tales como la participación de la respuesta inmunitaria innata que contribuye a activar la vía metabólica del triptófano regulada por la actividad de la enzima IDO, la cual interactúa con las reacciones del eje hipotálamohipófiso-suprarrenal y del sistema inmunitario innato. Pero, en contraste con las antiguas teorías sobre la depresión, que consideraban de manera aislada diferentes aspectos de la enfermedad, esta integra la participación de los factores señalados: el sistema serotoninérgico, su interacción con la vía de la quinurenina, el sistema adrenérgico, el eje hipotálamo-hipófiso-suprarrenal, y el equilibrio de los receptores NMDA/AMPA y los del tipo kainato, resaltando el papel de la modulación cruzada entre las vías de los TLR4, el IDO-glutamato y el eje hipotálamo-hipófiso-suprarrenal.

Como se ilustra en la figura $4 \mathrm{~A}$, la teoría más reciente propone que en las personas sanas se da la interacción equilibrada de los sistemas inmunitario y nervioso mediada por la actividad del eje hipotálamo-hipófiso-suprarrenal y por la relación entre la serotonina y la quinurenina, en la cual predomina la serotonina (tasa $>1$ ). En individuos propensos, esta relación se ve alterada cuando hay un exceso de estrés. En la figura 4B se muestra el incremento de la influencia del eje hipotálamo-hipófiso-suprarrenal y de la enzima IDO, lo cual reduce dicha tasa (tasa $<1$ ), y condiciona la hiperactividad del eje hipotálamohipofiso-suprarrenal y del sistema inmunitario, lo que, sumado a la hipoactividad del sistema nervioso, promueve la adaptación corporal al estrés y los cambios conductuales característicos de la enfermedad depresiva $(43,88,89)$.

Las interacciones entre estos sistemas ya han producido nuevas propuestas de tratamiento para la depresión mayor $(90,91)$.

\section{Conflicto de intereses}

Los autores declaran que no existe ningún conflicto de intereses relacionado con este manuscrito.
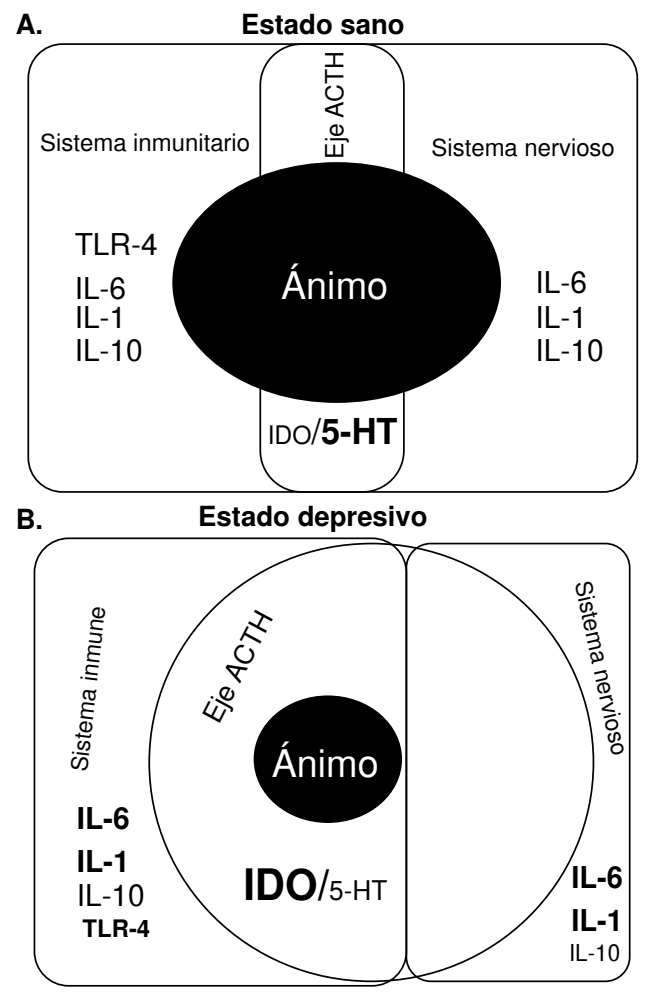

Figura 4. Interacción de los sistemas inmunitario y nervioso, y el eje hipotálamo-hipófiso-suprarrenal en el estado sano y en el depresivo. A. En el estado sano, las respuestas del sistema inmunitario y del nervioso se mantienen en equilibrio frente a la actividad del eje hipotálamo-hipofiso-suprarrenal y la relación entre la actividad de las enzimas IDO y la triptófano hidroxilasa. Dicho equilibrio produce un acoplamiento perfecto entre las áreas de influencia del sistema nervioso y las del inmunitario representadas por los óvalos que aparecen anclados en la actividad del eje hipotálamo-hipófiso-suprarrenal, y la relación de la actividad entre las enzimas indolamina 2,3-dioxigenasa (IDO) y triptófano hidroxilasa (TPH). B. En el estado depresivo, aumenta la actividad del eje hipotálamo-hipofiso-suprarrenal y de la vía de la quinurenina, así como los niveles de la enzima IDO y las interleucinas proinflamatorias IL-6 e IL-1b, en tanto que disminuye el de interleucinas antiinflamatorias como la IL-10, entre otras. En esta condición, las áreas del sistema inmunitario y del nervioso ya no se acoplan de manera perfecta y aparecen como dos áreas independientes.

\section{Financiación}

CONACYT CB2011-1674-Q a JCP; CB-2013-02 221653 a MA y CB-2011-168943 a HS.

\section{Referencias}

1. Sankowski R, Mader S, Valdes-Ferrer SI. Systemic inflammation and the brain: Novel roles of genetic, molecular, and environmental cues as drivers of neurodegeneration. Front Cell Neurosci. 2015;9:28. https://doi.org/10.3389/ fncel.2015.00028

2. Rosas-Ballina M, Olofsson PS, Ochani M, Valdés-Ferrer SI, Levine YA, Reardon C, et al. Acetylcholine-synthesizing $T$ cells relay neural signals in a vagus nerve circuit. Science. 2011;334:98-101. https://doi.org/10.1126/science.1209985 
3. Medzhitov R, Janeway CA Jr. Innate immunity: The virtues of a nonclonal system of recognition. Cell. 1997; 91:295-8. https://doi.org/10.1016/S0092-8674(00)80412-2

4. Jones JD, Dangl JL. The plant immune system. Nature. 2006;444:323-9. https://doi.org/10.1038/nature05286

5. Aballay A. Neural regulation of immunity: Role of NPR-1 in pathogen avoidance and regulation of innate immunity. Cell Cycle. 2009;8:966-9. https://doi.org/10.1016/S09609822(02)01396-9

6. Aballay A, Drenkard E, Hilbun LR, Ausubel FM. Caenorhabditis elegans innate immune response triggered by Salmonella enterica requires intact LPS and is mediated by a MAPK signaling pathway. Curr Biol. 2003;13:47-52. https://doi.org/10.1016/S0960-9822(02)01396-9

7. Maier SF, Watkins LR. Cytokines for psychologists: Implications of bidirectional immune-to-brain communication for understanding behavior, mood, and cognition. Psychol Rev. 1998;105:83-107. https://doi.org/10.1037/0033-295X. 105.1 .83

8. Maes $\mathbf{M}$, Song $\mathbf{C}$, Lin $\mathbf{A H}$, Bonaccorso $\mathbf{S}$, Kenis $\mathbf{G}$, De Jongh $\mathbf{R}$, et al. Negative immunoregulatory effects of antidepressants: Inhibition of interferon-gamma and stimulation of interleukin-10 secretion. Neuropsychopharma cology. 1999;20:370-9. https://doi.org/10.1016/S0893-133X (98)00088-8

9. Pace TW, Mletzko TC, Alagbe O, Musselman DL, Nemeroff CB, Miller AH, et al. Increased stress-induced inflammatory responses in male patients with major depression and increased early life stress. Am J Psychiatry. 2006;163: 630-3. https://doi.org/10.1176/ajp.2006.163.9.1630

10. Smith RS. The macrophage theory of depression. Med Hypotheses. 1991;35:298-306. https://doi.org/10.1016/03069877(91)90266-2

11. Hernández JC, Montoya CJ, Urcuqui-Inchima S. The role of toll-like receptors in viral infections: HIV-1 as a model. Biomédica. 2007;27:280-93. https://doi.org/10.7705/ biomedica.v27i2.225

12. Maes M, Smith R, Scharpe S. The monocyte-T-lymphocyte hypothesis of major depression. Psychoneuroendocrinology. 1995;20:111-6. https://doi.org/10.1016/0306-4530(94) 00066-J

13. Schiepers, OJ, Wichers MC, Maes M. Cytokines and major depression. Prog Neuropsychopharmacol Biol Psychiatry. 2005;29:201-17. https://doi.org/10.1016/j.pnpbp.2004.11.003

14. Capuron L, Gumnick JF, Musselman DL, Lawson DH, Reemsnyder A, Nemeroff CB, et al. Neurobehavioral effects of interferon-alpha in cancer patients: Phenomenology and paroxetine responsiveness of symptom dimensions. Neuropsychopharmacology. 2002;26:643-52. https://doi.org/S0893-133X(01)00407-9

15. Constant A, Castera L, Dantzer R, Couzigou P, de Ledinghen V, Demotes-Mainard J, et al. Mood alterations during interferon-alfa therapy in patients with chronic hepatitis C: Evidence for an overlap between manic/ hypomanic and depressive symptoms. J Clin Psychiatry. 2005;66:1050-7.

16. Ramírez K, Sheridan JF. Antidepressant imipramine diminishes stress-induced inflammation in the periphery and central nervous system and related anxiety- and depressivelike behaviors. Brain Behav Immun. 2016;57:293-303. https://doi.org/10.1016/j.bbi.2016.05.008

17. Raison CL, Capuron L, Miller AH. Cytokines sing the blues: Inflammation and the pathogenesis of depression. Trends Immunol. 2006;27:24-31. https://doi.org/10.1016/j. it.2005.11.006

18. Dantzer R, O'Connor JC, Freund GG, Johnson RW, Kelley KW. From inflammation to sickness and depression: When the immune system subjugates the brain. Nat Rev Neurosci. 2008;9:46-56. https://doi.org/10.1038/nrn2297

19. Cattaneo A, Macchi F, Plazzotta G, Veronica B, BocchioChiavetto L, Riva MA, et al. Inflammation and neuronal plasticity: A link between childhood trauma and depression pathogenesis. Front Cell Neurosci. 2015;9:40. https://doi. org/10.3389/fncel.2015.00040

20. Dantzer R, O'Connor JC, Lawson MA, Kelley KW. Inflammation-associated depression: From serotonin to kynurenine. Psychoneuroendocrinology. 2011;36:426-36. https://doi.org/ 10.1016/j.psyneuen.2010.09.012

21. O'Brien SM, Scott LV, Dinan TG. Cytokines: Abnormalities in major depression and implications for pharmacological treatment. Hum Psychopharmacol. 2004;19:397-403. https://doi.org/doi/10.1002/hup.609

22. Dantzer R. Cytokine-induced sickness behavior: Where do we stand? Brain Behav Immun. 2001;15:7-24. https://doi. org/10.1006/brbi.2000.0613

23. Leonard BE. The HPA and immune axes in stress: The involvement of the serotonergic system. Eur Psychiatry. 2005;20(Suppl.3):S302-6. https://doi.org/10.1016/S09249338(05)80180-4

24. González-Peña D, Nixon SE, O'Connor JC, Southey BR, Lawson MA, McCusker RH, et al. Microglia transcriptome changes in a model of depressive behavior after immune challenge. PLoS One. 2016;11:e0150858. https://doi. org/10.1371/journal.pone.0150858

25. Dantzer R, Bluthe RM, Laye S, Bret-Dibat JL, Parnet P, Kelley KW. Cytokines and sickness behavior. Ann N Y Acad Sci. 1998;840:586-90. https://doi.org/10.1111/j.17496632.1998.tb09597.x

26. Watkins LR, Wiertelak EP, Goehler LE, MooneyHeiberger K, Martínez J, Furness L, et al. Neurocircuitry of illness-induced hyperalgesia. Brain Res. 1994;639:28399. https://doi.org/10.1016/0006-8993(94)91742-6

27. Quan N, Whiteside M, Herkenham M. Time course and localization patterns of interleukin-1 beta messenger RNA expression in brain and pituitary after peripheral administration of lipopolysaccharide. Neuroscience. 1998; 83:281-93. https://doi.org/10.1016/S0306-4522(97)00350-3

28. Hasegawa-Ishii $S$, Inaba $M$, Umegaki $H$, Unno $K$, Wakabayashi K, Shimada A. Endotoxemia-induced cytokine-mediated responses of hippocampal astrocytes transmitted by cells of the brain-immune interface. Sci Rep. 2016;6:254-57. https://doi.org/10.1038/srep25457

29. Banks WA. The blood-brain barrier in psychoneuroimmunology. Neurol Clin. 2006;24:413-9. https://doi.org/10. 1016/j.ncl.2006.03.009 
30. Konsman J P, Vigues S, Mackerlova L, Bristow A, Blomqvist A. Rat brain vascular distribution of interleukin-1 type-1 receptor immunoreactivity: Relationship to patterns of inducible cyclooxygenase expression by peripheral inflammatory stimuli. J Comp Neurol. 2004;472:113-29. https://doi.org/10.1002/cne.20052

31. Dantzer R, Konsman JP, Bluthe RM, Kelley KW. Neural and humoral pathways of communication from the immune system to the brain: Parallel or convergent? Auton Neurosci. 2000;85:60-5. https://doi.org/10.1016/S1566-0702 (00)00220-4

32. Lenart N, Brough D, Denes A. Inflammasomes link vascular disease with neuroinflammation and brain disorders. J Cereb Blood Flow Metab. 2016;36:1668-85. https://doi.org/10.1177/0271678X16662043

33. Ericsson A, Kovacs KJ, Sawchenko PE. A functional anatomical analysis of central pathways subserving the effects of interleukin-1 on stress-related neuroendocrine neurons. J Neurosci. 1994;14:897-913. https://doi.org/10. $1002 / j n r .23550$

34. García-Oscos F, Peña D, Housini M, Cheng D, López D, Cuevas-Olguín R, et al. Activation of the anti-inflammatory reflex blocks lipopolysaccharide-induced decrease in synaptic inhibition in the temporal cortex of the rat. J Neurosci Res. 2015;93:859-65. https://doi.org/10.1002/jnr.23550

35. García-Oscos F, Peña D, Housini M, Cheng D, López D, Borland MS, et al. Vagal nerve stimulation blocks interleukin 6-dependent synaptic hyperexcitability induced by lipopolysaccharide-induced acute stress in the rodent prefrontal cortex. Brain Behav Immun. 2015;43:149-58. https://doi.org/10.1016/j.bbi.2014.07.020

36. Parnet P, Kelley KW, Bluthe RM, Dantzer R. Expression and regulation of interleukin-1 receptors in the brain. Role in cytokines-induced sickness behavior. J Neuroimmunol. 2002; 125:5-14. https://doi.org/10.1016/S0165-5728(02)00022-X

37. Tang MM, Lin WJ, Pan YQ, Guan XT, Li YC. Hippocampal neurogenesis dysfunction linked to depressive-like behaviors in a neuroinflammation induced model of depression. Physiol Behav. 2016:161:166-73. https://doi.org/10.1016/j. physbeh.2016.04.034

38. Vogelzangs $\mathbf{N}$, de Jonge $\mathrm{P}$, Smit JH, Bahn S. Penninx BW. Cytokine production capacity in depression and anxiety. Transl Psychiatry. 2016;6:e825. https://doi.org/10.1038/ tp.2016.92

39. Salgado H, García-Oscos F, Patel A, Martinolich L, Nichols JA, Dinh L, et al. Layer-specific noradrenergic modulation of inhibition in cortical layer II/III. Cereb Cortex. 2011;21:212-21. https://doi.org/10.1093/cercor/bhq081

40. Xu Y, Tao X, Shen B, Horng T, Medzhitov R, Manley JL, et al. Structural basis for signal transduction by the Toll/ interleukin-1 receptor domains. Nature. 2000;408:111-5. https://doi.org/10.1038/35040600

41. Watters TM, Kenny EF, O'Neill LA. Structure, function and regulation of the Toll//L-1 receptor adaptor proteins. Immunol Cell Biol. 2007;85:411-9. https://doi.org/10.1038/ sj.icb.7100095

42. Akira S, Takeda K. Toll-like receptor signalling. Nat Rev Immunol. 2004:4:499-511. https://doi.org/10.1038/nri1391

43. Cheng Y, Pardo M, Armini R de S, Martínez A, Mouhsine $\mathrm{H}$, Zagury JF, et al. Stress-induced neuroinflammation is mediated by GSK3-dependent TLR4 signaling that promotes susceptibility to depression-like behavior. Brain Behav Immun. 2016;53:207-22. https://doi.org/10.1016/j. bbi.2015.12.012

44. McCusker RH, Kelley KW. Immune-neural connections: How the immune system's response to infectious agents influences behavior. J Exp Biol. 2013;216:84-98. https://doi. org/10.1242/jeb.073411

45. Viviani B, Boraso M, Marchetti N, Marinovich M. Perspectives on neuroinflammation and excitotoxicity: $A$ neurotoxic conspiracy? Neurotoxicology. 2014;43:10-20. https://doi.org/10.1016/j.neuro.2014.03.004

46. Yeh KY, Shou SS, Lin Y X, Chen C C, Chiang C Y, Yeh CY. Effect of Ginkgo biloba extract on lipopolysaccharide-induced anhedonic depressive-like behavior in male rats. Phytother Res. 2015;29:260-6. https://doi.org/10.1002/ptr.5247

47. Remus JL, Dantzer R. Inflammation models of depression in rodents: Relevance to psychotropic drug discovery. Int J Neuropsychopharmacol. 2016;19:9. https://doi.org/10.1093/ ijnp/pyw028

48. Wichers MC, Maes $\mathbf{M}$. The role of indoleamine 2,3dioxygenase (IDO) in the pathophysiology of interferonalpha-induced depression. J Psychiatry Neurosci. 2004; 29:11-7.

49. Muller N, Schwarz MJ. The immune-mediated alteration of serotonin and glutamate: Towards an integrated view of depression. Mol Psychiatry. 2007;12:988-1000. https://doi. org/10.1038/sj.mp.4002006

50. Hashimoto $\mathbf{K}$. Emerging role of glutamate in the pathophysiology of major depressive disorder. Brain Res Rev. 2009;61:105-23. https://doi.org/10.1016/j.brainresrev.2009. 05.005

51. O'Connor JC, Andre C, Wang Y, Lawson MA, Szegedi SS, Lestage J, et al. Interferon-gamma and tumor necrosis factor-alpha mediate the upregulation of indoleamine 2,3-dioxygenase and the induction of depressive-like behavior in mice in response to bacillus Calmette-Guerin. J Neurosci. 2009;29:4200-9. https://doi.org/10.1523/ JNEUROSCI.5032-08.2009

52. Zunszain PA, Anacker C, Cattaneo A, Carvalho LA, Pariante CM. Glucocorticoids, cytokines and brain abnormalities in depression. Prog Neuropsychopharmacol Biol Psychiatry. 2011;35:722-9. https://doi.org/10.1016/j.pnpbp. 2010.04.011

53. Lestage J, Verrier D, Palin K, Dantzer R. The enzyme indoleamine 2,3-dioxygenase is induced in the mouse brain in response to peripheral administration of lipopolysaccharide and superantigen. Brain Behav Immun. 2002;16:596601. https://doi.org/10.1016/S0889-1591(02)00014-4

54. Frenois F, Moreau M, O'Connor J, Lawson M, Micon $\mathrm{C}$, Lestage $\mathrm{J}$, et al. Lipopolysaccharide induces delayed FosB/DeltaFosB immunostaining within the mouse extended amygdala, hippocampus and hypothalamus, that parallel the expression of depressive-like behavior. Psychoneuroendocrinology. 2007;32:516-31. https://doi. org/10.1016/j.psyneuen.2007.03.005

55. O'Connor JC, Lawson MA, Andre C, Moreau M, Lestage J, Castanon N, et al. Lipopolysaccharide-induced depressivelike behavior is mediated by indoleamine 2,3-dioxygenase activation in mice. Mol Psychiatry. 2009;14:511-22. https:// doi.org/10.1038/sj.mp.4002148 
56. Walker AK, Budac DP, Bisulco S, Lee AW, Smith RA, Beenders B, et al. NMDA receptor blockade by ketamine abrogates lipopolysaccharide-induced depressive-like behavior in C57BL/6J mice. Neuropsychopharmacology. 2013;38:1609-16. https://doi.org/10.1038/npp.2013.71

57. Gibney SM, McGuinness B, Prendergast C, Harkin A, Connor TJ. Poly I:C-induced activation of the immune response is accompanied by depression and anxiety-like behaviours, kynurenine pathway activation and reduced BDNF expression. Brain Behav Immun. 2013;28:170-81. https://doi.org/10.1016/j.bbi.2012.11.010

58. Fischer CW, Elfving B, Lund S, Wegener G. Behavioral and systemic consequences of long-term inflammatory challenge. J Neuroimmunol. 2015;288:40-6. https://doi. org/10.1016/j.jneuroim.2015.08.011

59. O'Connor JC, Lawson MA, Andre C, Briley EM, Szegedi SS, Lestage $\mathrm{J}$, et al. Induction of IDO by bacille CalmetteGuerin is responsible for development of murine depressivelike behavior. J Immunol. 2009;182:3202-12. https://doi. org/10.4049/jimmunol.0802722

60. Miller GE, Chen E, Sze J, Marin T, Arévalo JM, Doll R, et al. A functional genomic fingerprint of chronic stress in humans: Blunted glucocorticoid and increased NF-kappaB signaling. Biol Psychiatry. 2008;64:266-72. https://doi. org/10.1016/j.biopsych.2008.03.017

61. Hayden MS, West AP, Ghosh S. SnapShot: NF-kappaB signaling pathways. Cell. 2006;127:1286-7. https://doi. org/10.1016/j.cell.2006.12.005

62. Muller MB, Holsboer F. Mice with mutations in the HPAsystem as models for symptoms of depression. Biol Psychiatry. 2006;59:1104-15. https://doi.org/10.1016/j. biopsych.2006.02.008

63. Pace TW, Miller AH. Cytokines and glucocorticoid receptor signaling. Relevance to major depression. Ann N Y Acad Sci. 2009;1179:86-105. https://doi.org/10.1111/j.1749-6632. 2009.04984.x

64. Sapolsky RM, Meaney MJ, McEwen BS. The development of the glucocorticoid receptor system in the rat limbic brain. III. Negative-feedback regulation. Brain Res. 1985; 350:169-73.

65. Ulrich-Lai YM, Herman JP. Neural regulation of endocrine and autonomic stress responses. Nat Rev Neurosci. 2009; 10:397-409. https://doi.org/10.1038/nrn2665

66. Littrell JL. Taking the perspective that a depressive state reflects inflammation: Implications for the use of antidepressants. Front Psychol. 2012;3:297. https://doi.org/10.3389/ fpsyg.2012.00297

67. Zunszain PA, Anacker C, Cattaneo A, Choudhury S, Musaelyan K, Myint AM, et al. Interleukin-1beta: A new regulator of the kynurenine pathway affecting human hippocampal neurogenesis. Neuropsychopharmacology. 2012;37: 939-49. https://doi.org/10.1038/npp.2011.277

68. Pariante $\mathbf{C M}$, Miller AH. Glucocorticoid receptors in major depression: Relevance to pathophysiology and treatment. Biol Psychiatry. 2001;49:391-404. https://doi.org/10.1016/ S0006-3223(00)01088-X

69. Hennessy MB, Kaiser S, Sachser N. Stability and change: Stress responses and the shaping of behavioral phenotypes over the life span. Front Zool. 2015;12(Suppl.1):S18. https:// doi.org/10.1186/1742-9994-12-S1-S18
70. Anisman H, Zaharia MD, Meaney MJ, Merali Z. Do earlylife events permanently alter behavioral and hormonal responses to stressors? Int J Dev Neurosci. 1998;16:14964. https://doi.org/10.1016/S0736-5748(98)00025-2

71. Kanczkowski W, Alexaki VI, Tran N, Grossklaus S, Zacharowski K, Martinez A, et al. Hypothalamo-pituitary and immune-dependent adrenal regulation during systemic inflammation. Proc Natl Acad Sci U S A. 2013;110:14801-6. https://doi.org/10.1073/pnas.1313945110

72. Mohn CE, Fernández-Solari J, De Laurentiis A, Bornstein SR, Ehrhart-Bornstein M, Rettori V. Adrenal gland responses to lipopolysaccharide after stress and ethanol administration in male rats. Stress. 2011;14:216-26. https://doi.org/10.3109/10253890.2010.532254

73. Loum-Ribot E, Lafon P, Chaigniau M, Tramu G, Corio M. Glucocorticoids down-regulate lipopolysaccharide-induced de novo production of neurotensin mRNA in the rat hypothalamic, paraventricular, corticotrophin-releasing hormone neurons. Neuroimmunomodulation, 2006;13:170-8. https:// doi.org/10.1159/000098130

74. Gibb J, Al-Yawer F, Anisman H. Synergistic and antagonistic actions of acute or chronic social stressors and an endotoxin challenge vary over time following the challenge. Brain Behav Immun. 2013;28:149-58. https://doi. org/10.1159/000098130

75. Oliveira JF, Gomes CA, Vaz SH, Sousa N, Pinto L. Glial plasticity in depression. Front Cell Neurosci. 2016;10:163. https://doi.org/10.3389/fncel.2016.00163

76. Banasr M, Duman RS. Glial loss in the prefrontal cortex is sufficient to induce depressive-like behaviors. Biol Psychiatry. 200;64:863-70. https://doi.org/10.1016/j.biopsych. 2008.06.008

77. Cao X, Li LP, Wang Q, Wu Q, Hu HH, Zhang M, et al. Astrocyte-derived ATP modulates depressive-like behaviors. Nat Med. 2013;19:773-7. https://doi.org/ 10.1038/nm.3162

78. Sun JD, Liu Y, Yuan YH, Li J, Chen NH. Gap junction dysfunction in the prefrontal cortex induces depressive-like behaviors in rats. Neuropsychopharmacology. 2012;37: 1305-20. https://doi.org/10.1038/npp.2011.319

79. Di Benedetto B, Malik VA, Begum S, Jablonowski L, Gómez-González GB, Neumann ID, et al. Fluoxetine requires the endfeet protein aquaporin- 4 to enhance plasticity of astrocyte processes. Front Cell Neurosci. 2016;10:8. https://doi.org/10.3389/fncel.2016.00008

80. Czeh B, Simon M, Schmelting B, Hiemke C, Fuchs E. Astroglial plasticity in the hippocampus is affected by chronic psychosocial stress and concomitant fluoxetine treatment. Neuropsychopharmacology. 2006;31:1616-26. https://doi.org/10.1038/sj.npp.1300982

81. Etievant A, Lucas G, Dkhissi-Benyahya O, Haddjeri $\mathbf{N}$. The role of astroglia in the antidepressant action of deep brain stimulation. Front Cell Neurosci. 2016;9:509. https:// doi.org/10.3389/fncel.2015.00509

82. Kong $\mathrm{H}$, Sha LL, Fan $\mathrm{Y}$, Xiao M, Ding JH, Wu J, et al. Requirement of AQP4 for antidepressive efficiency of fluoxetine: Implication in adult hippocampal neurogenesis. Neuropsychopharmacology. 2009;34:1263-76. https://doi. org/10.1038/npp.2008.185 
83. Rial D, Lemos C, Pinheiro H, Duarte JM, Goncalves FQ, Real JI, et al. Depression as a glial-based synaptic dysfunction. Front Cell Neurosci. 2015;9:521. https://doi. org/10.3389/fncel.2015.00521

84. Sapolsky RM, Pulsinelli WA. Glucocorticoids potentiate ischemic injury to neurons: therapeutic implications. Science. 1985;229:1397-400. https://doi.org/10.1126/science.4035356

85. Eisenberger NI. The pain of social disconnection: Examining the shared neural underpinnings of physical and social pain. Nat Rev Neurosci. 2012;13:421-34. https://doi. org/10.1038/nrn3231

86. Slavich GM, O'Donovan A, Epel ES, Kemeny ME. Black sheep get the blues: A psychobiological model of social rejection and depression. Neurosci Biobehav Rev. 2010;35: 39-45. https://doi.org/10.1016/j.neubiorev.2010.01.003

87. Slavich GM, Cole SW. The emerging field of human social genomics. Clin Psychol Sci. 2013;1:331-48.

88. Frank MG, Thompson BM, Watkins LR, Maier SF. Glucocorticoids mediate stress-induced priming of microglial proinflammatory responses. Brain Behav Immun. 2012;26: 337-45. https://doi.org/10.1016/j.bbi.2011.10.005
89. Zacharowski K, Zacharowski PA, Koch A, Baban A,Tran N, Berkels R, et al. Toll-like receptor 4 plays a crucial role in the immune-adrenal response to systemic inflammatory response syndrome. Proc Natl Acad Sci USA. 2006;103:6392-7. https://doi.org/10.1073/pnas.0601527103

90. Hernández ME, Mendieta D, Pérez-Tapia M, Bojalil R, Estrada-García I, Estrada-Parra S, et al. Effect of selective serotonin reuptake inhibitors and immunomodulator on cytokines levels: An alternative therapy for patients with major depressive disorder. Clin Dev Immunol. 2013;2013: 267871. https://doi.org/10.1155/2013/267871

91. Arreola R, Becerril-Villanueva E, Cruz-Fuentes C, Velasco-Velázquez M A, Garcés-Álvarez M E, HurtadoAlvarado G, et al. Immunomodulatory effects mediated by serotonin. J Immunol Res. 2015;2015:354957. https://doi. org/10.1155/2015/354957 